Es ist anzunehmen, dafs aıch andere Molekularkrăfte, wie diejenigen, welche Elasticität und optische Eigenschaften der Körper bedingen, in ähnlicher Weise noch in endlicher Entfeınung wirlisam sind, und dürfle dieser Unstand die jetzt herrschenden theoretischen Ansichten über dieselben erheblich modificiren.

Berlin den 2. Mai 1869.

V. Ueber die Isodimorphie der arsenigen und der antimonigen Säure; von Paul Groth.

E ist bereits seit längerer Zeit bekannt, dafs die beiden Verbindungen $\mathrm{As}_{2} \mathrm{O}_{3}$ und $\mathrm{Sb}_{2} \mathrm{O}_{3}$ dimorph sind, und entweder in Formen des regulären oder in solchen des rhombischen Systems krystallisiren, und man hat wegen der vollständigen Analogie der Zusammensetzung beider die Isomorphie derselben auch in der zweiten der genannten Formen angenommen; bis jetzt sind jedoch keine Messungen der rhombischen Modification der arsenigen Säure bekannt gemacht worden, welche jene Isımorphie mit der als Weirsspiefsglanzerz in der Natur vorkommenden rhombischen antimonigen Säure bestätigen kőnnten. Vor einigen Jahren ist diese, immer nur selten bei metallurgischen Processen unter weiterhin zu besprechenden Umständen sich bildende rhombische arsenige Säure auf der Halsbrückener Hütte bei Freiberg von Neuem vorgehommen, und der Verfasser verdankt der Güte des Hrn. Th. Richter in Freiberg, dem er zu grofsem Danke für seine allzeit bereitwillige Gefälligkeit verpllichtet ist, die Uebersendung des Materials, welches ihn in den Stand gesetzl hat, die Krystallform jener Substanz eingehend zu untersuchen. Die rhombische arsenige Säure bietet eine reiche Entwickelung von Formen dar, 
welche wir vergebens bei dem entsprecbenden Antimonoxyd suchen; aber es ist dennoch möglich, beide Korper krystallographisch auf eine Grundform zu beziehen, sie also als isomorph zu betrachten, wcmn auch die Zahl ihrer gemeinschaftlichen Formen auf eine einzige, das Grundprisma, beschränkt ist. Im Folgenden sollen die Resultate jener Untersuchung, sowie einige andere Beobachtungen uber beide Körper mitgetheilt, endlich das Wichtigste über dieselben bereits Bekannte kurz zusammengestellt werden.

\section{Arsenige Säure.}

A) Reguläre.

Die gewöhnliche Form (Octaëder), in welcher sich die verflüchtigte arsenige $S$. absetzt, wenn die Temperatur des Raumes, in welchem diefs geschieht, keine zu hohe ist. Ebenfalls in regulären Oclaëdern (andere Formen scheinen nicht vorzukommen) scheidet sich dieselbe.aus Wasser, aus ammoniakalischer und aus salzsaurer Losung ab. Die auf trocknem Wege dargestellten Krystalle sind gewöhnlich undurchsichtig weifs, die aus Lösungen oft durchsichtig, nach Hrn. Hirzel (Zeitschr. f. Pharm. 1851, S. 81) besonders grofs beim Abkühlen einer heifs gesättigten Losung in Am. moniak zu erhalten.

Spec. Gewicht $=3,70$ bis 3,72 Karsten, $=3,699$ Guibourt.

Spec. Wärme $=0,1279$ Regnault, $=0,1309$ de la Rive und Marcet.

Brechungsexponent $=1,748$ Roth, $=1,755$ Gelb Des cloizea ur.

B) Rombische arsenige Säure.

Dafs der arsenigen Säure aufser der regulären anch eine rhombische Form zukommt, fand zuerst Hr. Wohler (d. Ann. Bd. XX VI, S. 177) bei Untersuchung eines Conglomerates von Krystallen, welche beim Abbruch eines Cobaltröstofens zu Schwarzenfels in Kurhessen gefunden worden waren. Dieselben waren farblos, durchsichtig, von sechssei- 
tiger Tafelform, wie Gyps sehr biegsam und nach der Hauptfläche leicht spaltbar. Er giebt ferner an, dafs m aus einer approximativen Messung Mitscherlich's fast mit Gewifsheit hervorgehe, dafs die Krystallform gleich der des Weifsspiefsglases sey r. Zur vollständigen krystallographischen Untersuchung scheinen dieselben nicht geeignet gewesen zu seyn, doch geht schon aus diesen Angaben hervor, dafs sie mit den weiter unten beschriebenen vollkommen identisch waren.

Einen sehr wichtigen Beitrag zur Kenntnịls dieser Substanz lieferte Hr. Pasteur (Ann. de pharm. et chim. [3], XIII, 399), indem er die Darstellung der in Rede stebenden Moditication auf nassem Wege kennen lehrte. Sättigt man siedende Kalilősung vollständig mit der Säure, so erhält ma eine syrupöse Flüssigkeit (saures arsenigs. Kalium), welche sich in Wasser löst, aber durch dessen Gegenwart allmählich zersetzt wird und einen Absatz von arseniger Säure bewirkı, welcher fast ohne Ausnahme in prismatischen Formen die $W$ ände des Glases bekleidet. $\mathbf{H r}$. Pasteur giebt die Uebereinstimmung dieser Formen mit denen des Weifsspiefsglanzes, und die Gleichheit des Anseheus beider, aber keine Messungen seiner Krystalle an, wozu dieselben jedenfalls zu klein waren. Der Verfasser hat die Pasteur'schen Versuche wiederholt und vollkommen bestätigt gefunden. Die in beschriebener Weise hergestellten Lösungen gaben in einem mälsig kühlen Raume in 1 bis 2 Wochen eine beträchtliche Menge des krystallisirten Niederschlags und zwar liefsen die seitlich an den Wănden ansitzenden gröfseren Krystalle bereits durch eine einfache Loupe dieselben Combinationen, wie sie weiterhin beschrieben werden sollen, einzelne sogar deutlich die flachen Pyramiden (s. unten die krystallogr. Beschreibung) erkennen, durch welche die Krystallform der arsenigen Säure so ausgezeichnet ist. Die prismatische Zone war weniger gut ausgebildet, doch liefsen sich diese Krystalle, von deren Gröfse Hr. Pasteur nichts angiebt, während Hr. Debray die Pasteur'schen Krystalle in seiner weiten unten zu erwähnenden Arbeit * mikroskopische* nennt, schon mit freiem 
Auge als Prismen erkennen. An Glanz und Ansehen waren sie, wie auch Hr. Pasteur angiebt, dem natürlichen Antimonoxyd sebr äbnlich. Leider war der Niederschlag nicht zur Bestimmung seiner sonstigen Eigenschaften (specif. Gewicht, Loslichkeit) zu gebrauchen, da er nicht vollständig von dewn auhängeuden syrupösen Salz zu trennen war. Dasselbe konnte nicht mit Wasser abgespült werden, da sich die Säure mit auflöste, noch weniger mit Alkohol, der das saure Salz ausfällt. In wenig Wasser beide gelöst, scheiden sie sich neben einander aus, leicht durch ihre Krystallform zu unterscheiden; die arsenige Säure in kurzen breiten Prismen, das saure arsenigsaure Kalium in sehr langen, dünnen, am Ende schief zugeschärften prismatischen Nadeln. Auch aus alkalischen, mit $\mathrm{As}_{2} \mathrm{O}_{3}$ nicht gesättiqten Lösungen in Kali scheidet diese sich in derselben Weise aus, nachdem sich bei langem Stehen an der Luft erst eine beträchtliche Menge Kryslalle von zweifach hohlensaurem Kalium abgesetzt hat. Die Koblensäure scheint dem basischeu und neutralen arsenigsauren Salze, welche, das eine in Anfang, das audere später, in der Flüssigkeit vorhanden seyn müssen, allmăhlich $K$ zu entziehen, während sich ein Niederschlag von $\mathrm{As}_{2} \mathrm{O}_{3}$ bildet, der dem aus sauren Lösungen erhaltenen ganz ähnlich ist.

Ebenfalls auf nassem Wege erhielten prismatische arsenige Säure Hr. Kühn (Archiv. d. Pharm. [2], LIX, 267) und Hr. Hirzel (Zeitschr. f. Pharmacie, 1851, 81), Ersterer aus einer Lösung von arsenigsaurem Silber in Salpetersăure in concentrischen Nadeln, Letzterer aus ammoniakalischer Lösung, welche unter Ammoniakzusatz längere Zeit zum Sieden- erhitzt wurde.

Nach der Past eur'schen Methode hat Hr. Nordenskjold (diese Ann. Bd. CXIV, S. 622) aus Kalilosung sechsseitige optisch zweiaxige Tafeln erhalten, welche er für Combinationen von $o P, \infty P, \infty \breve{P}_{\infty}$ genommen hat, und angiebt, dafs die Axen $a$ und $b$ sich verhielten $=1: 0,5776$ ungefähr (d. i. $=\cot 60^{\circ}$, also hat Derselbe wahrscheinlich

PoggendorfPs Annal. Bd. CXXXVIl. 
unter dem Mikroskop den ebenen Winkel der Tafel gemessen und ungefăhr $120^{\circ}$ gefunden). Ein Prisma von 120 an a giebt es aber bei der arsenigen Saure nicht, vielmehr ist oben angegeben worden, dafs dieselbe aus Kali stets in Prismen, welche nach $\infty \breve{P}_{\infty}$ breit tafelförmig sind, krystallisirt. Darnach ist es sehr wahrscheinlich, dafs die von Hrn. Nordenskjold erhaltenen Formen solche Tafeln nach $\infty \breve{P}_{\infty}$, begränzt durch ein Octaëder, waren. Endlich hat Hr. Scheurer-Kestner prismatische Nadeln von $\mathrm{As}_{2} \mathrm{O}_{3}$ erhalten, indem er eine concentrirle Lösung von Arsensäure mit arseniger Säure übersättiote (Bulletin de la soc. chim. Par. 1568, $X, 444)$.

Hr. Debray (Compt. rend. LVIII, 1209, Ann. d. Chem. und Pharm. Supplbd. III, 250) hat durch einen wichligen Versuch die Bedingungen kennen gelehrt, unter welchen sich die Dämpfe der arsenigen Säure in der prissnatischen Form niederschlagen. Er hat amorphe Säure in ein Glasrohr eingeschlossen und dieses, mit einer passenden Hülle umgeben, um sein schnelles Erkalten zu verhüten, vertical über einem Gasofen angebracht und 8 bis 10 Stunden lang erhilzt, wobei der untere Theil eine Temperatur von $400^{\circ}$, der obere nur von $200^{\circ}$ annahın. Nach dem Erkalten befand sich unten noch etwas glasige Säure, in dem mittleren Theile des Rohres eine grofse Menge bereits mit freiem Auge erkennbarer Prismen, im oberen nur Octaëder; im Verlauf der Abkühlung hatten sich auf die Prismen einzelne Oclaëder aufgesetzl. Es ist durch diesen schönen Versuch vollkommen festgestellt, dafs die fragliche Substanz sich auf solchen Wänden, welche beträchtlich heilser sind, als $200^{\circ}$, stets in der rhombischen Modification absetzt, und dadurch auch das verhältnifsmäfsig seltene Vorkommen derselben bei metallurgischen Processen erklärt, da in den Flugstaubkammern, wo die aus den Oefen tretenden Gase zuerst Gelegenheit zu ruhiger und allmähliger Verdichtung (der Bedingung für das Entstehen deutlicher Krystalle) finden, die Wande selten eine so hohe Temperatur haben durften. 
Die wenigen Fălle, in denen die rhombische arsenige Säure seit der ersten Wahrnehmung durch Hrn. Wöbler auf trocknem Wege als zufällig entstanden wieder beobachtet worden ist, sind die folgenden:

Hr. F. Ulrich beobachtete (Zeitschr. f. d. gesammt. Naturwiss. von Giebel und Heintz, XI, 261) auf der Okerhütte am Harz arsenige Säure, welche sich in Rösthaufen auf einzelnen Erzstïcken (die also eine besonders hohe Temperatur angenommen hatten) in dünnen rechtwinkligen Tafeln abgesetzt hatte, welche am freien Ende sehr spitz zugeschärft erschienen. Dieselben waren aber nicht biegsam und spaltbar, sondern zeigten im Bruch deutlich unter dem Mikroskop, dafs sie aus einem Aggregat kleiner Octaëder bestanden, also Paramorphosen der regulären nach der rhombischen $\mathrm{As}_{2} \mathrm{O}_{3}$ waren. Es ist nicht bekannt, was hier den Uebergang der einen in die andere Moditication bedingt habe. Hr. Ulrich theilt mir brieflich mit, dafs schon vor etwa 20 Jahren glattllächige glănzende, also nicht paramorphosirte Krystalle unter ähnlichen Verhältnissen atuf der Okerhütte rorgekommen seyen.

Wahrscheinlich das massenhafteste aller bisherigen Vorkommnisse ist das von Hrn. F. Claudet beschriebene (Journal of the Chemic. Soc. Lond. 1868, p. 179) aus den Schwefelkiesgruben von San Domingos in Portugal. Daselbst hat das, durch den neuen Grubenbetrieb erfolgte, Trockenlegèn der von den Römern herrührenden und mit Wasser erfüllt gewesenen alten Baue eine spontane Entzündung der darin aufgehäuften Kiesmassen hervorgerufen, das in den Erzen enthaltene Arsen ist in grofsem Maafsstab verflüchtigt worden und hat sich in den höher befindlichen Spalten und Höhlungen in, bis ein Zoll langen, rhombischen Krystallen abgesetżt. Dieselben batten alle von Hrn. Wöhler angegebenen Eigensehaften, waren halbdurchsichtig, hatten ein spec. Gewicht von 3,85 , eine Härte von $2 \frac{1}{2}$, und zeigten Einwirkung auf das polarisirte Licht. Kaltes Wasser löste von den Krystallen 1 Proc., von den gepulverten 1,75 Proc; eine gesăttigte siedende Lösung enthielt 2,47 Proc., 
also etwa so viel, als sie octaëdrische arsenige Säure aufzunehmen vermag. Durcb Sublimation und Lösung gaben sie nur Octaëdcr.

Einen dem vorigen sehr ähnlichen Fall theilt Hr. Scheurer-Kestner (Bull. d. l. soc. çhim. Par. 1868, t. X, 444) mit. In dem Hauptkanal eines Kiesofens, welcher die schweflige Säure in die Bleikanmern führt, hatten sich mehrere Millimeter lange, grad rhombische Prismen von $\mathrm{As}_{2} \mathrm{O}_{3}$ abgeselzt. Hr. Sch.K. sagt a. a. O., dafs in den vou Wöhler, Claudet und ihm beschriebenen Fällen die Verbindung sich stets in einem langsamen Strome von schwefliger Säure niedergeschlagen habe, und hält diefs für eine wesentliche Beding̨ung der Bildung der rhombischen Modification. Sein Versuch, dieselbe im Kleinen unter diesen Umständen herzustellen, ist indefs vergeblich gewesen, während andrerseits Hr. Debray (s. oben) nachgewiesen hat, dafs sie sich ohne schweflige Säure in hoher Temperatur bildet. Wenn anch demnach jene Vermuthung des Hrn. Scheurer-Kest ner nicht richtig, und die erste Bedingung für ihre Entstebung die geeignete Temperatur ist, so bleibt doch dabei nicht ausgeschlossen, dafs vielleicht auch das zugleich vorhandene schwefligsaure Gas eine Einwirkung in dieser Richtung haben kann.

In allen genannten Fällen scheint die Substanz wicht in solchen Krystallen vorgekommen zu seyn, welche sich zu Messungen geeignet hätten. Diefs ist nur der Fåll mit denjenigen, welche i. J. 1867 auf der Halsbrückner Hütte bei Freiberg in Sachsen gefunden worden sind. Nach einer kurzen Mittheilung ihres Vorkommens (Berg - und Huttenmänn. Zeitung, 1867, S. 143) wurden sie beim Einreifsen eines Schachtofens innerhalb der Wände des Ofens, etwa 1 Fufs von der Innenfläche und ebenso viel uber der Form gefunden, während in gröfserer Höhe über derselben sich nur Oclaëder abgesetzt hatten. Die Iämpfe der arsenigen Säure hatten also die porösen Wände des Ofens durchdrungen und sich in den Höhlungen da, wo die Temperatur die erforderliche Höhe hatte, in der prismatischen, weiterhin 
in der gewöhnlichen Form niedergeschlagen. Da die Poren demnach mit einander communicirten, könuen Differenzen des Druckes, wie a. a. O. vermuthet ist, nicht mitgewirkt haben, sondern nur solche der Temperatur, ganz wie in dem beschriebenen J ebray'schen Versuch in dem Glasrohr offenbar überall derselbe Druck herrschen mufste. Das erwăhnte, vielleicht das schönste der bisherigen Vorkommnisse, ist leider erst etwas zu spät beachtet worden, so dafs nur eine ganz kleine Portion des interessanten Körpers gesammelt und in der Sammlung des Oberhüttenamts zu Freiberg niedergelegt worden ist. Trotzdem hatte $\mathrm{Hr}$. Th. Richter, welcher derselben vorsteht, die Gïte, mir so viel davon zur Verfügung zu stellen, als zu einer eingehenderen Untersuchung nöthig war, deren Resultate nun in dem Folgenden mitgetheilt werden sollen:

Beschreibung der rhombischen arsenigen Säure von Freiberg. Die farblosen Krystalle sitzen dichtgedrängt theils direct nuf der Ofenmasse, theils auf einer fasrigen und mehligen Unterlage, die aus derselben Substanz besteht, welche wahrscheinlich schnellerem Absatz ihre Entsiehung verdankt und daher nicht deutlich krystallisirt erscheint. Ihre Gröfse geht bei einzelnen tafelförmigen bis zu 12 Millim. Länge, 5 Millim. Breite und I bis 2 Millim. Dicke, indefs sind die wohl ausgebildeten, auf welche sich die Abbildungen (Fig. 1 bis 4 Taf. VII) beziehen, kleiner, 4 bis 5 Millim. lang und etwa 2 Mllm. breit. Es sind rhombische Prismen, durch Vorherrschen der Abstumpfung der scharfen Kanten breit tafelförmig '). An dem freien Ende ist fast immer das primäre Octaëder $o$ am gröfsten ausgebildet, daneben eine Reihe sehr flacher Pyramiden $\alpha, \beta, \gamma$, welche sämmtlich mit einander und mit der Tafelfläche $b$ in einer Zone liegen, s. Fig. 1 und 3 Taf. VII. Oft sind von den beiden Zonen $b, \alpha, \beta, \gamma$ dieses Poles nur die eine deutlich ausgebildet (Fig. 4), während von den Flächen der anderen nur geringe

1) Vergl, die Figuren 1 bis 4 Tafel VII, welche die complicirteren Combinationen darstellen, und Fig. 5, die eine Projection sämmtlicher Flächen nach der $Q$ uenstedt'schen Methode gieht. 
Andeutungen vorhanden sind, so dafs ein solcher Krystall ein monoklinisches Ansehen erhält, oder, da die Schiefe der Kanten $\gamma: \gamma$ gegen die Horizontale nur unbedeutend ist, diese Octaëder leicht für rhombische Domen gehalten werden könnten. Selten ist eine dieser Zonen ganz allein am freien Ende ausgebildet. Wenn das Octaëder $o$ vorherrscht, ist stets seine Combinationskante mit $b$ durch ein anderes, $n$, abgestumpft. Alle genaunten Flächen sind zwar klein, aber von hellstem Glanze, so dafs selbst diejenigen, welche nur kleine Bruchtheile eines Quadratmillimeters grofs waren, mit ziemlicher Annäherung gemessen werden konnten. Anders verhält es sich mit der verticalen Zone, die sich zwar ebenfalls reich an Flächen zeigte, welche aber sämmtlich so matt, gestreift und gekrümmt erschienen, dafs nur sehr ungenaue Bestimmungen derselben ermöglicht werden konnten, daher bei einigen das Zeichen unbestimmt blieb. Die vorbandenen Flächen, mit Einschluf́s dieser nicht sicher zu bestimmenden, sind die folgenden:

\begin{tabular}{|c|c|c|c|c|}
\hline (Buchst. d. Fig.) & & (Naumann) & & (Weifs) \\
\hline 0 & $=$ & $P$ & $=$ & $(a: b: c)$ \\
\hline$n$ & $=$ & $\bar{P} \frac{1}{7}$ & $=$ & $\left(a: \frac{1}{7} b: c\right)$ \\
\hline$\alpha$ & $=$ & $\frac{1}{12} \bar{P}_{4 \overline{8}}^{\frac{1}{4}}$ & $=$ & $\left(12 a: \frac{1}{4} b: c\right)$ \\
\hline$\beta$ & $=$ & $\frac{1}{12} \bar{P}_{24}^{\frac{1}{24}}$ & $=$ & $\left(12 a: \frac{1}{2} b: c\right)$ \\
\hline$\gamma$ & $=$ & $\frac{1}{1 \overline{2}} \overline{\boldsymbol{P}}_{\overline{1} \overline{2}}$ & $=$ & $(12 a: b: c)$ \\
\hline$\delta$ & $=$ & $\frac{1}{12} \bar{P} \infty$ & $=$ & $(12 a: \infty b: c)$ \\
\hline$a$ & $=$ & $\infty \bar{P}_{\infty}$ & $=$ & $(a: \infty b: \infty c)$ \\
\hline $\boldsymbol{b}$ & $=$ & $\infty \breve{P}_{\infty}$ & $=$ & $(\infty a: b: \infty c)$ \\
\hline$p$ & $=$ & $\infty P$ & $=$ & $(a: b: \infty c)$ \\
\hline$m$ & $=$ & $\infty \bar{P}_{2}$ & $=$ & $\left(\frac{1}{2} a: b: \infty c\right)$ \\
\hline$[\mu$ & $=$ & $\infty \bar{P}_{\overline{5}}$ & $=$ & $\left.\left(\frac{5}{2} a: b: \infty c\right)\right] ?$ \\
\hline$[v$ & $=$ & $\infty \bar{P}_{\frac{1}{5}}$ & $=$ & $(5 a: b: \infty c)] ?$ \\
\hline$[z$ & $=$ & $x P$ & $=$ & $(a: b: x c)] ?$ \\
\hline
\end{tabular}

Von diesen Flächen liefs sich das Grundoctaëder $o$ am genauesten bestimmen, daher die an demselben erhaltenen 
Messungsresultate der Rechnung zu Grunde gelegt wurden; indefs weichen auch bier, mehr noch bei den ubrigen Formen, die an verschiedenen Krystallen gewonnenen Zahlen weit mehr von einander ab, als die Beschaffenheit der meist ganz ebenen Flächen erwarten liefse. So wurde für $o: o$ (Kante $A$ ) an verschiedenen Krystallen gefunden $97^{\circ} 19^{\prime}-27^{\prime}$, besser ubereinstimmend $o: b=104^{\circ} 20^{\prime}-23^{\prime}$. Für diese beiden Winkel wurden die Mittel der zuverlässigsten Werthe zur Rechnong genommen. $n$ war stets so schmal, dafs es nur an zwei Krystallen annkhernd gemessen werden konnte, doch genügten die erbaltenen Zahlen zur Bestimmung seines Zeichens. $\delta$ wurde nur an einem Krystall als sehr schmale Abstumpfung der Endkante $\gamma: y$ gefunden, welche Flache gegen $b$ ungefähr $9^{\circ}$, wie die Messung ergab, geneigt war. $a$ ist ebenfalls sehr selten, wenigstens als mefsbare Fläche; angedeutet erscheint es allerdings stets durch die Rundung der stumpfen Prismenkante. Wie bereits oben bemerkt, ist die Ausbildung der prismatischen Zone eine sehr ungünstige. Fig. 2 giebt eine Andeutung der Lage der Flächen derselben. Das Grundprisma $p$, obgleich nur schwach spiegelnd, konnte durch seine Neigung gegen $o$ (s. unten den Werth i. d. Tabelle) sicher erkannt werden, da es in dieser Richtung nicht gekrümmt ist, sondern nur von rechts nach links, nach den benachbarten prismatischen Flächen hin. Aus diesem letztern Grunde wurde aber seine Neigung gegen $b=\infty \breve{P}_{\infty}$ von $107^{\circ}$ bis $113^{\circ}$ gefunden (berechnet: $110^{\circ} 36^{\prime}$ ). Sicher bestimmen liefs sich nur einmal ein anderes Prisma $m$ (s. Tab.). Aufser diesen sind noch zwei schärfere vorhanden, deren Zeichen indefs nicht mit voller Sicherheit zu bestimmen waren. Die Neigung des einen, $\mu$, zu $b$ wurde im Mittel einiger, um mehrere Grade abweichenden, Bestimmungen zu $131^{\circ} 5 \mathrm{I}^{\prime}$ gefunden; das Zeichen $\infty \bar{P}_{5}^{2}$ würde $133^{\prime \prime} 13^{\prime}$ erfordern. Für das zweite $\nu$ ergab sich ebenso $154^{\circ} 23^{\prime}$ ' (berechnet: $151^{\circ} 56^{\prime}$ für $\infty \bar{P}_{\frac{1}{5}}$ ). Endlich liegt noch nahezu in der prismatischen Zone ein sehr steiles Octaëder $z$ der Hauptreihe, ungefär $a: b: 15 \mathrm{c}$. Dasselbe ist fast immer 
vorhanden (vielleicht sogar mehrere), oft ziemlich vorherrschend (s. Fig. 2, 3, 4) und giebt zuweilen den Krystallen das Ansehen, ăhnlich dem spiefsig krystallisirten Aragonit. Diese Flächen sind aber stets so matt und zusammengesetzt, dafs keine zuverlässigen Messungen zu erhalten waren, überdiefs sind sie sehr leicht mit Scheinflächen zu verwechseln, welche durch das Aneinanderlegen benachbarter Individuen entstanden.

Im Folgenden sind die sämmtlichen Resultate der Messungen zusammengestellt. Es bedentet dabei $a$ die Brachydiagonale, $b$ die Makrodiagonale, $c$ die Verticalaxe; $A$ die makrodiagonale Polkante, $B$ die brachydiagonale Polkante, $C$ die horizontale Basiskante der rhombischen Pyramiden. Für die Rechnung sind die mit * bezeichneten Werthe (s. dariber oben) genommen, und neben den daraus berechneten Neigungswinkeln ist jedesmal, wo ein Winkel nur an einem Krystall mit Sicherheit ermittelt werden konnte, dieser Werth allein als "beobachtet" angegeben, andernfalls das Mittel der zuverlässigsten Bestimmungen an mehreren Krystallen; letztere Zahlen sind mit M. (= Mittel) bezeichnet.

Axenverhältni/s:

Neigungswinkel:

$$
a: b: c=0,3758: 1: 0,3500 \text {. }
$$

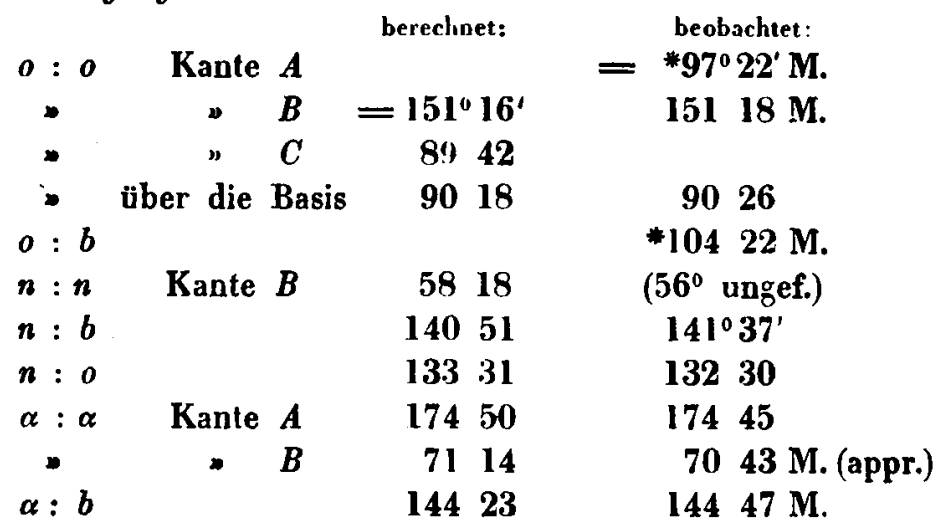




\section{5}

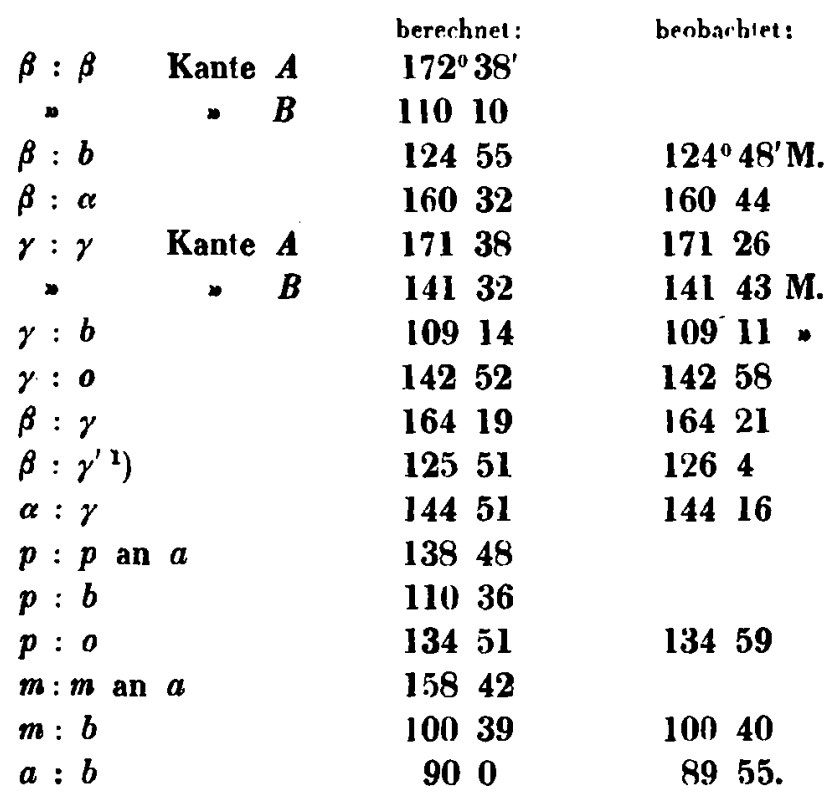

Spaltbar sind die Krystalle, wie schon Hr. Wöhler angegeben, nach $b=\infty P \infty$ mit ausgezeichneter Vollkommenheit, so dafs man die dünnsten und biegsamsten Blättchen nach dieser Fläche ablösen kann. Ferner existirt ein fasriger Bruch wach dem Prisma $p$. Wegen der sehr grofsen Weichheit und Biegsamkeit, welche die des Gypses übertrifft, ist letzterer schwer darzustellen.

Optische Untersuchung. Die optischen Eigenschaften liefsen sich, theils wegen der Kleinheit der meisten Krystalle, besonders aber wegen der Schwierigkeit des Schleifens einer so weichen und biegsamen Substanz, senkrecht auf den Hauptblätterbruch, der dem des Glimmers Nichts nachgiebt, nur sehr unvollständig bestimmen. Die optische Axenebene ist die vollkommne Spaltfläche $\propto \breve{P}_{\infty}$, von den in dieser Ebene liegenden Elasticitälsaxen ist die Hauptaxe die der kleinsten, die Brachydiagonale die der gröfsten Elasticität. Von den normal zu jener Fläche angelegten Schliffen gelang aus den

1) Die an der anderen Seite liegende Fläche von $\gamma$, gemessen über $\delta$. 
bereits angegebenen Gründen nur derjenige senkrecht zur verticalen Axe so weit, um in Luft einige undeutliche Farbenringe zu zeigen, welche in Oel mit Natriumlicht zwar mehr hervortraten, ohne indefs so deutliche Axenbilder zu liefern, dafs die Platte zur genauen Messung des Axenwinkels tauglich gewesen wäre. Der Winkel der oplischen Axen ergab sich als nahe einem rechten gleich; wahrscheinlich ist also die vertikale Axe $c$ die erste Mittellinie, und, da diese die Axe kleinster Elasticität ist, die Krystalle negativ.

Die Abweichung der optischen Eigenschaften von denen des isomorphen Antimonoxyds, welche Hr. Descloizeaux (Nouv. recherch. s. l. propr. opt. d. crist. Par. 1867, p. 58) milgetheilt, sind sehr erheblich.

Das specifische Gewicht der rhombischen $\mathrm{As}_{2} \mathrm{O}_{3}$ wurde in absolutem Alkohol (wegen der Löslichkeit in Wasser), dessen spec. Gew. in zwei Versuchen zu 0,79567 und 0,79535 bestimmt wurde, genommen und daraus dasjenige in Bezug auf Wasser berechnet. Es ergab sich zu 4,151 (ein weniger genauer Controllversuch 4,217). Diese Zahl ist so viel höher, als die von Hrn. Claudet gefundene (s. S. 419) von 3,85, dafs die Substanz des Letzteren wahrscheinlich schon theilweise paramorphosirt gewesen seyn muls. Bei der arsenigen Säure ist demnach, wie beim Antimonoxyd, das spec. Gew. der rhombischen Modification höher, als das der regulären, wie folgende Uebersicht zeigt:

$$
\begin{array}{lcr} 
& \mathrm{As}_{2} \mathrm{O}_{3} & \mathrm{Sb}_{2} \mathrm{O}_{3} \\
\text { Regulär : } & \mathbf{3 , 7 0} & \mathbf{5 , 2 6} \\
\text { Rhombisch : } & \mathbf{4 , 1 5} & \mathbf{5 , 5 7}
\end{array}
$$

\section{Antimonige Săure}

A) Reguläre.

Während bei der arsenigen Säure die octaëdrische Form die gewöhnlich sich bildende, die prismatische die weit seltnere ist, verbält sich die analoge Sauerstoffverbindung des Antimons bekanntlich umgekehrt. Sie krystallisirt genöhnlich in Formen des rhombischen Systems. Reguläre Octaëder hat nach Mitscherlich (d. Ann. Bd. XXVI, S. 180) 
zuerst von Bonsdorff dargestellt, indem er Antimonchlorid in kohlensaurem Natrium löste, wobei sich das Oxyd in der genannten Form abschied. Hr. Wöhler erwähnt aufserdem a. a. O., dafs Zinken anf der Harzgeroder Hütte beim Schmelzen von Schwefelantimon Octaëder von $\mathrm{Sb}_{2} \mathrm{O}_{3}$ beobachtet, und er selbst durch auhaltendes Schmelzen von Antimon dergleichen dargestellt habe. Hr. Pasteur, der von Bonsdorff's Versuch nicht gekannt zu haben scheint, hat diese Modification in ganz analoger Weise, wie jener, erhalten (Ann. d. pharm. et chim. [3], t. XIII, p. 399). Er hat frisch gefälltes und mit uberschüssiger koblens. Natrium. lobsung ausgewaschenes Algarolbpulver melirere Tage lang mit jener digerirt; das entstandene bräunliche Oxyd zeigte unter dem Mikroskop Octaëder (zuweilen auch Cubooctaëder), daneben aber auch prismatische Krystalle. Hr. Debray (a. a. O.) giebt an, dafs die Auflusung von Antimonoxydhydrat in Kalilauge beim Erkalten kleine Octaëder liefere, und dafs (nach Mitscherlich) besonders grofse zu erzielen seyen, wenn man eine Losung von $\mathrm{Sb} \mathrm{Cl}_{3}$ in $\mathrm{HCl}$ mit heifsem Wasser versetzt, bis der Niederschlag sich eben nicht wieder auflöst, und erkalten läfst.

Viel ausgezeichneter und gröfser krystallisirt findet sich nun, bisher aber nur an zwei Fundorten, das octaëdrische Antimonoxyd in der Natur als Senarmontit, in der Provinz Constanline in Algier. und bei Perneck in Ungarn. Von diesem Mineral theilt Hr. Descloizeaux (Nouv. rech. 8. $l$. propr. opt. d. crist. Par. 1867, p. 9) mit, dafs die Krystalle desselben ähnliche Erscheinungen darbieten, wie sie derselbe Beobachter an dem Boracit gefnnden hat. Platten, senkrecht zu den Octaëderaxen geschliffen, zeigen bei parallelem Licht im Polarisationsapparat unregelmắsige Farben und eine mehr oder weniger vollstăndige Auslöschung in vier triangulären Sectoren, welche durch schmale gekrilmmte Banden, die aufeinander rechtwinkelig und den Würfelflächen parallel sind, getrennt werden. Das convergirende Licht lafst hie und da ein Ringsystem, durchsechnitten von einer schwarzen Bande und verschieden orientirt je nach dor untersuchten Platte, 
erkennen. Dasselbe scheine einer optischen Axe, die etwas schief zur Platte steht, zu entsprechen. Obgleich die Ringe im Allgemeinen breiter, weniger zahlreich und mehr verwaschen, als im Boracit, seyen, so meint doch Hr. Descloizeaux, dafs die Erscheinung ohne Zweifel von der Einlagerung zahlreicher, doppeltbrechender Lamellen einer andern Substanz herrühre, während es wenig wahrscheinlich sey, dafs es die Wirkung unregelmäfsiger Polarisation sey. Da die eingelagerte Substanz kein Weifsspiefsglanzerz, das zwei sehr nahe liegende optische Axen besitzt, seyn kann, vermuthet er darin rhombische arsenige Säure, da der Senarmontit oft Spuren von Arsen enthält. Allerdings ist durch die oben mitgetheilten Beobachtungen erwiesen, dafs die rhombische arsenige Säure zwei sehr divergirende Axen besitzt, doch scheint mir die Doppelbrechung derselben nicht so stark zı seyn, dafs sie in so dünnen Lamellen, in denen sie hier anzunehmen wäre, noch derartige Farbenringe zeigen könne. Auch erscheint das Zusammenkrystallisiren der rhombischen Modification der cinen mit der octaëdrischen der andern Säure chemisch ziemlich unwahrscheinlich, vielmehr liegt es näher, anzunehmen, dafs der geringe Arsengehalt als isomorphe Beimischung (regulär) darin vorhanden ist. Die Beobachtungen Hrn. Descloizeaux's und einige eigne darüber scheinen mir auf eine andere Erklärung hinzudeuten. Durch die octaëdrische Spaltbarkeit der Sub$\left.\operatorname{stan} z^{1}\right)$ ist es leicht, sehr kleine und dünne, ganz durcbsichtige Spaltungsblättchen zu erhalten; diese unter einem stärkern Mikroskop untersucht, liefsen keine fremdartige Einlagerung, oft aber eine eigenthümliche Zusammensetzung und Streifung nach den Octaëderflächen erkennen. Verband man unit dem Mikroskop eine polarisirende Vorrichtung, so zeigten klare derartige Splitter überall gleichmäfsig eine ganz regelmäfsige zweimalige Auslöschung beim Drehen, vollstăndig wie ein zweiaxiger Krystall, oboleich ibre Gestalt sie deutlich als gespaltene Octaëdersegmente kennzeichnete. Andere Stückchen waren durch eine deutliche Gränze in

1) Dieselbe wurde mir gütigst von $\mathrm{Hrn}$. G. Rose zur Verfügung gesteltt. 
zwei Theile geschieden, in welchen die Lage der optischen Hauptschnitte und auch die oberflächliche Streifung eine andere war. Diese Erscheinungen erinnern so auffallend an die im Alaun, von weichem Hr. Reusch (diese Ann. Bd. 132, S. 618) nachgewiesen hat, dafs er in den Octaëdersectoren durch Spannungen, die jedenfalls bei dem Act der Krystallisation eingetreten sind, in sehr regelmäfsiger Weise doppeltbrechend ist, so dafs es der Verfasser für wahrscheinlicher hält, dafs dieselbe Ursache auch die eigenthümlichen. Erscheinungen, welche der Sénarmontit darbietet, bedinge Damit würde vor Allem auch die Beobachtung des Hrn Descloizeaux selbst über die Auslöschung in vier triangulären Sectoren, welche ganz analog der Erscheinung am Alaun ist (s. Reusch a. a. O.), übereinstimmen. Was die im Polarisationsmikrosk op sichtbaren Farbenringe betriff, so miifste diese dann in derselben Weise, wie in geprefsten und gekühlten Gläsern, entstehen, womit ihr Ansehen nach den Beobachtungen, die der Verfasser angestellt hat, nicht im Widerspruch zu stehen scheint.

Der Brechungsexponent des Sénarmontit ist $=2,073$ Roth, 2,087 Gelb nach Descloizeaux.

Das spec. Gero. $=5,22-5,30$.

B) Rhombisclie antimonige Säure.

In dieser Form tritt dieselbe gewöhnlich auf beim Erhitzen von Antimon unter Luftzutritt, wo sich, wie bekannt, der Regulus beim Erkalten mit kleinen glänzenden Nadeln umkleidet. Derselben gebört ferner der Niederschlag an, der sich bei allmählichem Zusatz von kohlensaurem Natrium zu einer Lossung von $\mathrm{Sb} \mathrm{Cl}_{3}$ in Salzsäure bildet (Mitscher lich). Wie bei der arsenigen Säure, hat Hr. Debray (a. a. O.) auch für die antimonige nachgewiesen, dafs sich die prismatische besonders in böherer Temperatur bildet, indem er fand, dafs die aus sauren, wie aus alkalischen Lösungen oberhalb $100^{\circ}$ erhaltene Säure stets die rhombische, wie fast immer anch die durch die Wărme allein dargestellte, ist. 
Endlich ist die rhombische Form anch diejenige, in wolcher gewobnlich das Antimonoxyd in der Natur (als Wei/sspie/sglanserz, Antimonblüthe, Exitèle) auftritt. Die Krystallform der Antimonblïthe hat bereits 1824 Mohs (Mine* ralogie II, 168) kennen gelehrt. Er führt als vorkommende Flächen auf: ein Prisma $M$ von $1^{136^{\prime \prime}}$ 58, ein nicht genau mefsbares brachydiagonales Doma $p$, dem er den Endkantenwinkel $70^{\circ} 32^{\prime}$ (den des regulären Octaëders) zuschreibt, die Abstumpfung der Combinationskanlen beider durch ein Octaëder $P$, endlich das Brachypinakoïd $h$. Das Octaëder $P$ nimmt er zur Grundform ') und giebt ihm das Axenverhältnifs $a$ (Verticale) $: b: c=10: \vee 12,5: \vee 7,77$. Da er für dieses so wenig, als für das Doma, wirkliche Messungen angiebt, mufs man die Form der Antimonblitbe als nur unvollständig bekannt ansehen. Die Angaben von Mohs sind in allen spätern Handbiichern der Mineralogie unverändert wiedergegeben, mit Ausnahme desjenigen von Hrn. Breith a up $t$, welcher die hypothetische Gröfse $109^{\circ} 28^{\prime}$ dem Endkantenwinkel, nicht wie Mohs, dem Seitenkantenwinkel des Domas zuschreibt. Hr. Dana (i. d. letzten Aufl. seiner Mineralogie S. 184) giebt ebenfalls keine neuen Messungen, nimmt aber das von Mohs bestimmte Prisma $M$ zum Grundprisma $\infty P$, dessen Doma $p$ als $\breve{P}_{\infty}$; dadurch erbält er das Axenverhältnifs $a: b: c=0,3943: l: 1,4141 \quad(=1: 2,5365$ $: 3,5868$ ). Iie von Mohs beobachteten Formen erhalten dann die Zeichen: $M=\infty P, \quad h=\infty \breve{P}_{\infty}, \quad p=\breve{P}_{\infty}$, $P($ Mohs $)=2 \breve{P}$ 2. Aufserdem führt Hr. Dana als beobachtete Flächen noch $\frac{1}{3} \bar{P}_{\infty}$ und $4 \check{P}_{\infty}$ auf, ohne über ihr Vorkommen oder etwaige Messungen derselben etwas mitzutheilen. Hr. Descloizeaux giebt (Nouv. rech. p. 58) für das Weifsspiefsglanzerz den Prismenwinkel von Mobs, $136^{\circ} 58^{\prime}$, und die Spaltbarkeit nach diesem Prisma und uach $\infty \breve{P}_{\infty}$ an, während Mohs die Absonderung nach der letztern Fläche nicht als Spaltbarkeit, sondern als eine Folge von Zusam-

1) In diesem Falle erhält das $\mathbf{P r i s m a}_{\boldsymbol{M}}$ das Zeichen $\infty \overline{\mathbf{P}} 2$ und das Doma p d. Z. $\frac{1}{2} \breve{p}_{\infty}$. 
mensetzung aus mehreren Individuen ansah, was indefs nicht begründet zu seyn scheint. Hr. Descloize aux beschreibt die braunen Krystalle ron Bräunsdorf als keilförmige Octaëder, bestehend aus dem glattgerundeten Doma ${ }_{3}^{1} \breve{P}_{\infty}$ oder $\frac{1}{4} \breve{P}_{\infty}$; für die weifsen, nach $\infty \breve{P}_{\infty}$ tafelförmigen Krystalle von Przibram und Sempsa (Provinz Constantive) giebt er an, dafs sie manchmal das Doma ${ }_{3}^{4} \breve{P}_{\infty}$ zeigen. Auch hier sind keine neuen Messungen mitgetheilt. Von den durch Hrn. Descloizeaux's Beschreibung so genau charakterisirten Krystallen von Bräunsdorf habe ich ein in dem hiesigen königl. Mineraliencabinet befindliches Exemplar gemessen. Das Prisma gab in Mittel $137^{\circ} 42^{\prime}$, doch waren die Flächen gestreift und lieferten keine einfachen Bilder. Die brachydomatischen Flächen waren so stark gekrümmt und so matt, dafs eine Messung ganz unmöglich war. Indefs liefs sich erkennen, dafs zwei Domen vorhanden waren, von denen das eine nach schälzungsweiser Messung, mittelst des Eintretens des Lichtschimmers auf den Flächen, einen Eudkantenwinkel von ungefähr $110^{\prime \prime}$ besafs, das andere flacher war. Bei Annahme des Axenverhältnisses, dessen sich Hr. Dana und wahrscheinlich auch Hr. Descloize a ux bediente, würde dem ersteren also wohl das Zeichen $\frac{1}{2} \breve{P} \propto$, letzterem vielleicht $\frac{1}{3} \breve{P} \infty$ oder $\frac{1}{4} \breve{P}_{\infty}$ zukommen. Diese Krystalle entsprechen ferner vollkommen der Figur und Beschreibung, welche Hr. Breithaupt (Lehrb. d. Mineral. Bd. II, S. 185 und Fig. 194) von den Bräunsdorfer Krystallen giebt, der sie als Combination eines Prismas von 136" $58^{\prime}$ und eines Brachydoma von $109^{\circ} 28^{\prime}$ Endkantenwinkel beschreibt. Man sieht hieraus, dafs unsere Kenntnil's der Krystallform der Antimonblüthe keineswegs eine genaue und vollsländige ist, und es daher sehr wünschenswerth wäre, mit möglichst guten Krystallen, wie sie dem Verfasser nicht zu Gebote standen, eine Revision derselben vorzunehmen ${ }^{1}$ ).

1) Hr. Pastenr hat zuerst die Bernerkung gemacht (Ann. chim. phys. t. XXIII, p. 276), dafs in dimorphen Körpern fast immer solche Winkel vorkommen, welche gewissen der andern Form sehr nahe stehen. 
Spec. Gewicht $=5,566$ Mohs.

Die optischen Eigenschaften s. Descloizeaux, Nouv. rech. s. l. propr. opt. etc., 58.

Vergleichung der Krystallformen der rhombischen arsenigen und antimonigen Säure.

Wie aus dem bereils Mitgetheilten hervorgeht, haben die beiden in Rede stchenden Substanzen, deren Isomorphie ihrer so vollig analogen Constitution halber keinem $Z_{w}$ eifel unterliegen kann, nur eine einzige gemeinschaftliche Krystallgestalt, das Prisma von $137^{\circ} 42^{\prime}\left(136^{\prime \prime} 58^{\prime}\right.$ Mohs) der Antimonblüthe und das von $138^{\circ} 48^{\prime}$ der arsenigen Säure. Es ist also passend, dieses, nach welchem auch bei beiden die Spaltbarkeit geht, zum Grundprisma beider Krystallreihen zu nehmen. Zur primăren Pyramide empliehlt sich in der reichen Formenreihe, welche der letztgenannte der beiden Körper aufzuweisen hat, die von uns gewäblte $o$ durch ihr stetés Vorherrschen, durch die verhältnifsmäfsig gröfsere Einfachheit der auf sie bezogenen Zeichen der andern Gestallen, durch ihre Mittelstellung zwischen den sehr flachen Pyramiden $(\alpha, \beta, \gamma)$ einerseits und den sebr steilen $(n, z)$ andererseits, endlich dadurch, dafs sie éine der zugehörigen zil jenem, als primäres zu betrachtenden Prisma ist. Darnach ist das Axenverhältnifs der arsenigen Säure, wie bereits angegeben:

$$
a: b: c=0,3758: 1: 0,3500 \text {. }
$$

Beziehen wir nun die am Weifsspiefsolanzerz beobachteten Flächen auf ein entsprechendes Axenverhältnifs, so miissen die von Mohs angegebenen folgende Zeichen erhalten: $M=\infty P, p=4 \breve{P} \infty, P=4 \bar{P}_{2}^{1}, h=\infty \breve{P} \infty$. Das von Hrn. Breitha upt als primäres angegebene Brachydoma von $109^{\circ} 28^{\prime}$ Endkantenwinkel wird dann $2 \breve{P} \infty$, die von Hrn. Da na als $\frac{1}{3} \breve{P}_{\infty}$ und $4 \dot{P}_{\infty}$ angeführten Domen wer-

Diels ist auch der Fall bei dem Antimonoxyd, wo der Winkel eines Doma dem Octaëderwinkel so mahe stcht, dals Mohs ihn demselben gleich gesetzt hát. 
den resp. $\frac{1}{3} \breve{P}_{\infty}$ uud $16 \breve{P}_{\infty}$, endlich das von Hrn. Des cloizeaux und von mir als ungefähr $\frac{1}{\dddot{q}} \breve{P}_{\infty}$ oder $\frac{1}{4} \breve{P}_{\infty}$ angegebene wird $\frac{1}{3} \breve{P}_{\infty}$ oder $\breve{P} \propto$. Mit Zugrundelegung der nenen Messung des Prisina zu $137^{\circ} 42^{\prime}$ und der von Mohs herrübrendeu hypothetischen Annahme des Winkels $4 \breve{P}_{\infty}$ : $4 \breve{P} \infty=70^{\prime \prime} 32^{\prime}$, für welche noch kein Ersatz durch eine erneute Messung geliefert worden ist, berechnet sich das Grundparameterverhältnifs der Antimonblüthe zu:

$$
a: b: c=0,3869: 1: 0,3710 \text {, }
$$

welches, zusammen mit dem damit zu vergleichenden der rhombischen arsenigen Süure als ein vorläufiger Ausdruck der Isomorphie der beiden Körper dienen hann, his neue Ụntersuchun:en das zweite der beiden Verbältnisse genauer festgestellt haben werden.

Berlin, Mai 1869.

\section{Ueber Kryslallform und Circularpolarisation und über den Zusammenhang beider beim Quarz und ïberjodsauren Natrium; von Paul Groth.}

(Aus den Mouatsber, d. Berl. Akad. d. Wissensch. Febr. 1869 vom Verf. mitgetheilt.)

$E_{s}$ ist bekannt, dafs inan bisher unter den einaxigen circularpolarisirenden Krystallen am Quarz allein mit Sicherheit solche Krystallfächen kennt, welche den Sinn der Drehung der Polarisationsebene des Lichtes vorher zu bestimmen erlauben. Es sind diefs die Trigonoëder (trigonalen Pyramiden), welche bei rechtsdrehenden Krystallun die Kante des Hauptrhomboëders, wenn der Beobachter eine obere Fläche desselben vor sich hat, init der zur rechten Seile liegenden Prismenfläche abstumpfen; ferner die Trapezoëder, von welcheu zweierlei Arten unterschiecien werden müssen, welche

Poggendorf's Annal. Bd. CXXXVIl. 
Rhomb. arsenige Säure

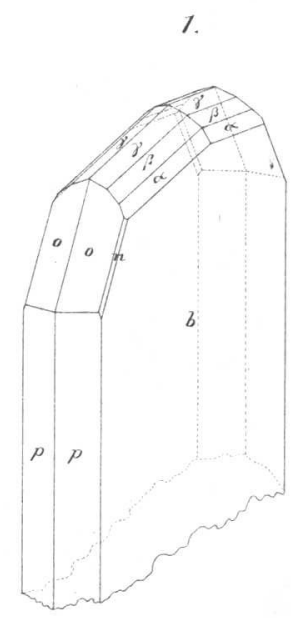

2.
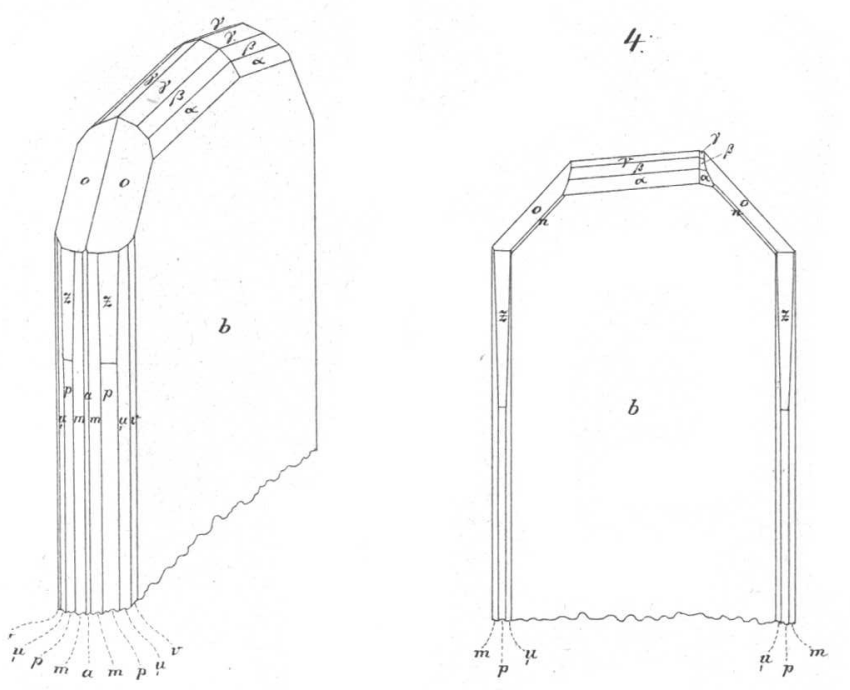

T. Grothy ael.

c. Sogriveze Sirtf. Ënst. Oberlin.

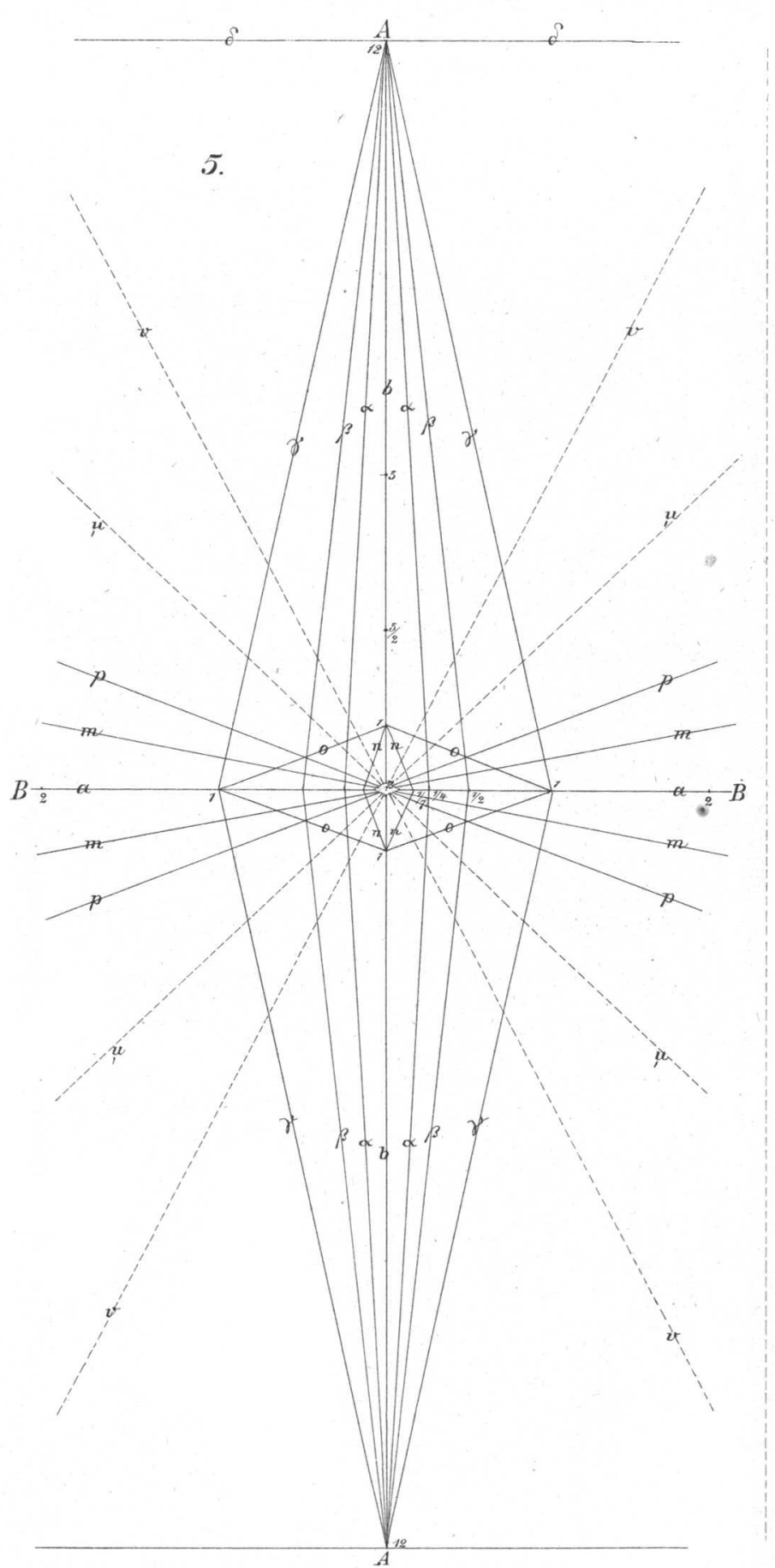

Quar: u. überjods. Natr:
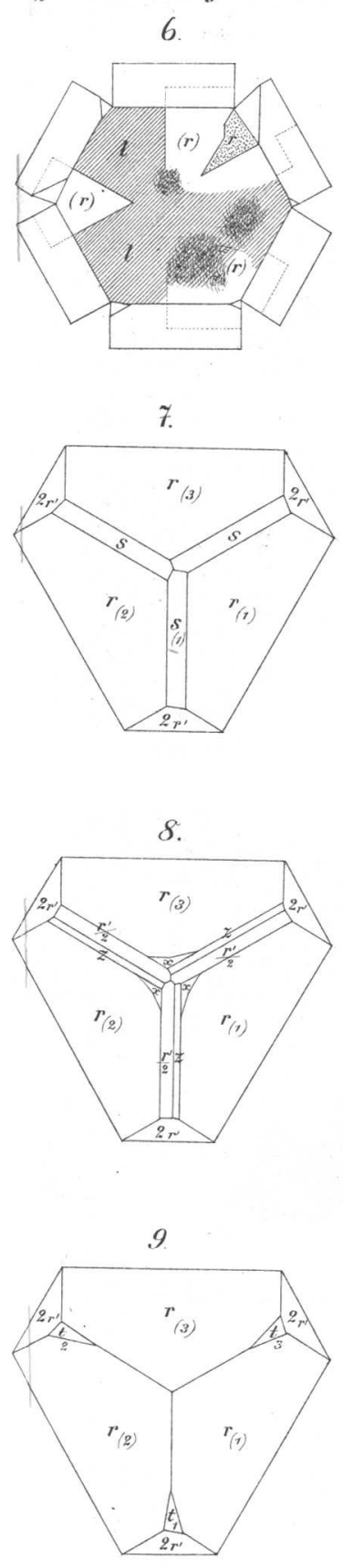

Taf VII

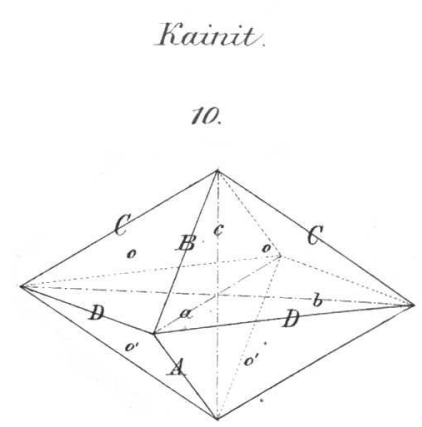

11
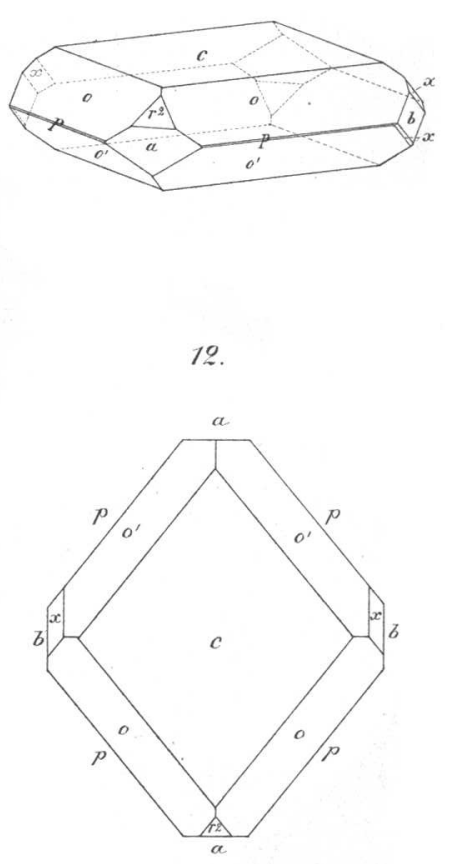

Ann. d. Phys. u. Chem. Bd. CXXXVIT. St. 3 